\title{
Radiographic evaluation of mandibular bone remodeling by mandibular cortical Index (MCI) in a Senegalese population
}

\section{NDIAYE Mamadou Lamine ${ }^{1, *}$, LECOR Pape Abdou², DIATTA Mamadou ${ }^{3}$, VOYEMANDE Augustin ${ }^{4}$, TOURE Babacar ${ }^{5}$}

\author{
1,2,3,4 Assistant Professor, ${ }^{5}$ Professor, ${ }^{1,4}$ Service of Dento-Maxillofacial Radiology, ${ }^{2}$ Service of Oral Physiology, ${ }^{3}$ Service of Oral \\ Surgery, ${ }^{5}$ Service of Conservative Odontology, Dept. of Odontology, Cheikh Anta Diop University of Dakar, Senegal
}

\author{
*Corresponding Author: NDIAYE Mamadou Lamine
}

Email: mlndiaye04@gmail.com

\begin{abstract}
The objective of this study was to evaluate radiographic changes of the mandible by mandibular cortical index (MCI) within a Senegalese population.

Materials and Methods: The study was performed on digital panoramic radiographs of Senegalese melanoderm subjects. The radiographs were classified into 3 age groups: 18-40 years (Group I), 41-55 years (Group II), 56 years and over (Group III). The MCI was ranked according to Klemetti in three scores (C1, C2 and C3). The score was assigned by observing the morphology of the lower mandibular cortex. The chi-square test was used to study the relationship of MCI with age and sex. The level of significance was set at $\mathrm{p} \leq 0.05$.

Results: A total of 82 numerical radiographs were selected. In men, 79.07\% ( $\mathrm{n}=34)$ had normal cortex $(\mathrm{C} 1)$ and $69 \%(\mathrm{n}=27)$ in women. The $\mathrm{C} 2$ variant was more common in women $(\mathrm{n}=10)$ and the $\mathrm{C} 3$ score was only found in women. The appearance of the lower cortex of the mandible was significantly associated with age $(\mathrm{p}=0.009)$.

Conclusion: This study has shown that MCI is a sensitive tool for the diagnosis of bone changes in the mandible in black African populations. However, these assertions should be confirmed by other studies with more representative samples.
\end{abstract}

Keywords: Bone remodeling, Panoramic x-ray, Mandible, Radiography digital, African population.

\section{Introduction}

With age, the loss of bone density can be identified by the reduction of the radio-opacity of the bone, thinning and porosity of cortices, or trabecular configuration changes on X-ray. ${ }^{1-3}$ The orthopantomogram providing an image of the entire mandible and maxilla ${ }^{4}$ allows the detection of bone changes. Mandibular changes are related to age, dental status and other factors. ${ }^{5-7}$ The orthopantomogram is a routine examination in the dental office. Panoramic radiomorphometric indices have a potential value in the detection of structural changes in the maxillary and mandible. ${ }^{8,9}$ Several studies have reported the contribution of panoramic radiomorphometric indices in the diagnosis of osteoporosis. These studies were performed in Caucasian and Asian populations. ${ }^{10-12}$ At the current state of knowledge there are no studies on the contribution of panoramic radiomorphometric indices in the diagnosis of osteoporosis concerning black African populations. However, inter-ethnic anatomical variabilities constitute a problem of applicability of standardized panoramic radiomorphometric values to black African populations.

The objective of this study was to evaluate the radiographic changes in the mandible using mandibular cortical index (MCI) according to Klemetti's classification $^{12}$ on panoramic X-ray in a Senegalese population.

\section{Materials and Methods}

The study was performed on digital panoramic radiographs of Senegalese melanoderm subjects. X-rays were taken during routine dental examinations of patients aged 18 years and older. Panoramic X-rays were collected at an imaging centre in Dakar from April to June 2016. The images were obtained using a CRANEX Novus e (B panoramic radiograph (Sodorex ${ }^{\circledR}$ ) by a single operator.

The inclusion criteria of the study included clear, high-quality, and gross distortion-free x-ray images of patients with good general health that provided good reading of anatomical structures: the mandible body, the mental foramen, the lower border of the mandible, the angle of the mandible and the condyles.

The socio-demographic data (age, sex and date of radio recording) was recorded along with the panoramic radiographs. X-rays were classified into 3 groups based on age:

1. $18-40$ years old (Group I)

2. 41-55 years old (Group II)

3. 56 years old and over (Group III)

$\mathrm{X}$-rays were analyzed by two examiners, a dental surgeon who is specialized in dento-maxillofacial radiology and a radiologist. The evaluation method was standardized. The images were examined on a computer screen in JPEG format. To avoid inter and intraexaminer variability, calibration was first performed on 30 random radiographs. Interrogator reproducibility was calculated by re-evaluating 15 randomly selected panoramic radiographs. Cohen's kappa was calculated for the degree of concordance. 
The mandibular cortical index (MCI) was given by Klemetti [12] (Fig. 1):

$\mathrm{C} 1$ : normal cortex, the endosteal margin of the cortex is still visible and strong on both sides;

C2: cortex eroded slightly or moderately, the endosteal margin showed semilunar defects (lacunary resorptions) where it appears for cortical residues;

C3: cortical layer severely eroded.
The scoring was done by observing the morphology of the lower mandibular cortex.

Statistical analysis of the data was performed by SPSS 20 software. The results were expressed in number and percentage. The chi-square test was used to study the relationship between MCI and age or sex. The level of significance was set at $\mathrm{p} \leq 0.05$.

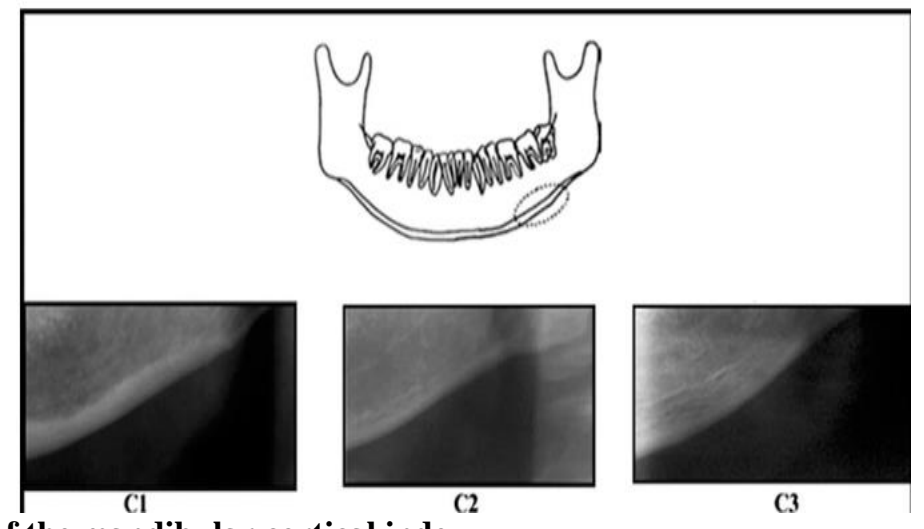

Fig. 1: Representation of the mandibular cortical index

Table 2: The distribution of the MCI by sex

\section{Results}

A total of 82 panoramic radiographs were selected and analyzed. The age group 18-40 was relatively more represented at $45.12 \%(n=37)$, followed by those aged 56 and over with $31.71 \%$ of the sample $(n=26)$ (Table 1). The sex ratio was 1:1. For inter-examiner agreement, the kappa value was 0.87 (good agreement).

In males, $79.07 \%(\mathrm{n}=34)$ had a normal cortex $(\mathrm{C} 1$ index) and $69 \%(n=27)$ in females had a $\mathrm{C} 1$ type MCI. The mildly or moderately eroded cortex (type C2 MCI) was found in $17 \%$ of males $(n=19)$ and $25.64 \%$ of females $(n=10)$. Finally the severely eroded cortical layer $(\mathrm{C} 3$ type $\mathrm{MCI})$ was only found in $5.12 \%(\mathrm{n}=2)$ females. Bone changes were more noticeable in females compared to males; but the difference was not statistically significant $(\mathrm{p}=0.2)$ (Table 2).

The distribution of MCI scores by age group was presented in Table 3. Among the three cortical-like scores, the $\mathrm{C} 1$ score was the most common in all age groups, However, its frequency decreased with age: $40 \%(n=33)$ in Group I and $17.08 \%(n=14)$ in Group III. The C2 score was more frequent in Group III, at $12.2 \%(\mathrm{n}=10)$. The C3 score was found only in women in Group III with $2.44 \%(\mathrm{n}=2)$. The appearance of the lower cortex of the mandible was significantly associated with age $(\mathrm{p}=0.009)$.

Table 1: Age distribution of the sample

\begin{tabular}{|l|c|c|}
\hline AGE (years) & $\mathbf{N}$ & \% \\
\hline Group I & 37 & 45.12 \\
\hline Group II & 19 & 23.17 \\
\hline Group III & 26 & 31.71 \\
\hline Total & 82 & 100 \\
\hline
\end{tabular}

\begin{tabular}{|l|c|c|c|c|c|}
\hline & \multicolumn{2}{|c|}{ Males } & \multicolumn{2}{c|}{ Females } & \\
\hline MCI & $\mathbf{N}$ & $\mathbf{\%}$ & $\mathbf{N}$ & $\boldsymbol{\%}$ & $\boldsymbol{p}$ \\
\hline C1 & 34 & 79.07 & 27 & 69.23 & \multirow{2}{*}{0.2} \\
\hline C2 & 9 & 20.93 & 10 & 25.64 & \\
\hline C3 & 0 & & 2 & 5.12 & \\
\cline { 1 - 5 } Total & 43 & 100 & 39 & 100 & \\
\hline
\end{tabular}

Table 3: MCI distribution by age

\begin{tabular}{|l|c|c|c|c|c|c|c|}
\hline $\begin{array}{c}\text { Age } \\
\text { (years) }\end{array}$ & $\begin{array}{c}\text { C1 } \\
\text { N }\end{array}$ & \% & $\begin{array}{c}\text { C2 } \\
\text { N }\end{array}$ & \% & $\begin{array}{c}\text { C3 } \\
\text { N }\end{array}$ & \% & p \\
\hline Group I & 33 & 40 & 4 & 4.88 & 0 & & \\
\hline Group II & 15 & 18.29 & 4 & 4.88 & 0 & & 0.009 \\
\hline Group III & 14 & 17.08 & 10 & 12.2 & 2 & 2.44 & \\
\hline Total & 62 & 75.62 & 18 & 21.96 & 2 & 2.44 & \\
\hline
\end{tabular}

\section{Discussion}

Bone tissue is subject to constant changes due to bone remodelling processes. Thus, the mandible undergoes various morphological changes that are influenced by the dental status and age of the subjects. ${ }^{13}$ Klemetti's MCI method has been used in many studies that have shown its interest in the study of bone changes in the mandible. ${ }^{1-3,5-18}$ This study conducted on African melanoderm population, was aimed at evaluating associations between bone changes, age and sex by mandibular cortical indices on panoramic radiography. This was a preliminary study conducted on a Senegalese population. It was based on 82 radiographs of Senegalese subjects, with a sex ratio of 1:1 which shows a good distribution of the study population according to gender. The results of this study proved that CMI is related to age and sex. These results are in line with those of the literature. A study Kiswanjaya et al., ${ }^{11}$ over 9 years of 280 Japanese women aged 70 years at baseline, showed a significant 
relationship between MCI and bone calcium in women. Female subjects were 3.26 times more likely to have lower mandibular erosion than male subjects. ${ }^{11}$ The $\mathrm{C} 1$ score in our study was higher in males than in females, which is similar to some previous studies. ${ }^{14}$ According to the study by Bajoria et al. $70 \%$ of subjects under 40 years old were having $\mathrm{C} 1$ score and $30 \%$ were with $\mathrm{C} 2$ score. ${ }^{1} \mathrm{C} 2$ and $\mathrm{C} 3$ scores were more prevalent among women over 40 years of age. This result could be explained by the higher osteoclastic activity leading to of bone resorption in women due to hormonal changes (deficiencies in estrogen) related to menopause and osteoporosis. ${ }^{5,15}$ The increased tonicity of jaw muscles result in excessive forces can accelerate bone resorption and loss of teeth. In a study by Gulsahi et al, patients with the type of MCI C3 should be considered at high risk of osteoporosis regardless of age and gender. ${ }^{16}$ Ferreira et al. considered MCI to be one of the specific indices in the evaluation of low bone mineral density (BMD). ${ }^{17}$ Khojastehpon et al showed sensitivity and specificity of $80.7 \%$ and $69.4 \%$ respectively with a positive predictive value of $70.7 \%$ of the MCI compared to the BMD of the lumbar vertebrae evaluated at bone densitometry in women at menopause. ${ }^{18}$ Kiswanjaya et $\mathrm{al}^{11}$ and Hastar et $\mathrm{al}^{14}$ concluded that the MCI could be used as an alternative way to evaluate low BMD of the skeleton. However, some authors did not find any correlations between gender and MCI. ${ }^{16,8}$

This study has shown that radiomorphometric indices, in particular MCI is a sensitive and specific tool for the diagnosis of bone changes including osteoporosis in melanoderm populations. However, these claims should be confirmed by other studies with more representative samples. Like other medical specialties, the dental surgeon can participate in the early detection of osteoporosis and prevent the risk of bone fractures using panoramic dental X-rays.

\section{References}

1. Bajoria AA, Ml A, Kamath G, Babshet M, Patil P, Sukhija P. Evaluation of Radiomorphometric Indices in Panoramic Radiograph - A Screening Tool. The Open Dentistry Journal. 2015;9:303-10.

2. Devlin CV, Horner K, Devlin H. Variability in measurement of radiomorphometric indices by general dental practitioners. Dentomaxillofac Radiol. 2001;30:120-5.

3. Dutra V, Devlin H, Susin C, Yang J, Horner K, Fernandes AR. Mandibular morphological changes in low bone mass edentulous females: evaluation of panoramic radiographs. Oral Surg Oral Med Oral Pathol Oral Radiol Endod. 2006;102:663-8.

4. Foucart JM, Felizardo R, Pizelle C, Bourriau J. Indications for radiography in orthodontics and dentofacial orthopedics. Orthod Fr. 2012;83(1):59-72.

5. Bozdag G, Sener S. The evaluation of MCI, MI, PMI and GT on both genders with different age and dental status. Dentomaxillofacial Radiology. 2015;44(9):1-9.

6. Dutra V, Susin C, da Costa NP, Veeck EB, Bahlis A, Fernandes A da RC. Measuring cortical thickness on panoramic radiographs: a validation study of the Mental Index. Oral Surg Oral Med Oral Pathol Oral Radiol Endod. nov 2007;104(5):686-91.

7. Horner K, Devlin H. The relationship between mandibular bone mineral density and panoramic radiographic measurements. J Dent. 1998;26:337-43.

8. Ledgerton D, Horner K, Devlin H, Worthington H. Panoramic mandibular index as a radiomorphometric tool: an assessment of precision. Dentomaxillofac Radiol. 1997;26:95-100.

9. Muramatsu C, Horiba K, Hayashi T, Fukui T, Hara T, Katsumata A, et al. Quantitative assessment of mandibular cortical erosion on dental panoramic radiographs for screening osteoporosis. Int J Comput Assist Radiol Surg. 2016;11(11):2021-32.

10. Dutra V, Yang J, Devlin H and al. « Radiomorphometric indices and their relation to gender, age, and dental status. Oral and maxillofacial radiology. 2005;99(4):479-84.

11. Kiswanjaya B, Yoshihara A, Deguchi T, Hanada N, Miyazaki H. Relationship between the mandibular inferior cortex and bone stiffness in elderly Japanese people. Osteoporos Int. 2010;21:433-8.

12. Klemetti E, Kolmakov S, Kroger H. Pantomography in assessment of the osteoporosis risk group. Scand J Dent Res. 1994;102:68-72.

13. Dutra V, Yang J, Devlin H, Susin C. Mandibular bone remodelling in adults: evaluation of panoramic radiographs. Dentomaxillofac Radiol. 2004;33:323-8.

14. Hastar E, Yilmaz HH, Orhan H. Evaluation of mental index, mandibular cortical index and panoramic mandibular index on dental panoramic radiographs in the elderly. Eur J Dent. 2011;5:60-7.

15. Nakajima $S$, Osato $S$. Association of gonial angle with morphology and bone mineral content of the body of the adult human mandible with complete permanent dentition. Ann Anat. 2013;195:53-8.

16. Gulsahi A, Paksoy CS, Ozden S, Kucuk NO, Cebeci ARI, Genc Y. Assessment of bone mineral density in the jaws and its relationship to radiomorphometric indices. Dentomaxillofac Radiol. 2010;39(5):284-9.

17. Ferreira Leite A., De Souza Figueiredo PT, Ramos Barra F., Santos de Melo N., de Paula A.P. Relationships between mandibular cortical indexes, bone mineral density, and osteoporotic fractures in Brazilian men over 60 years old. Oral Surg. Oral Med. Oral Pathol. Oral Radiol Endod. 2011;112(5):648-56.

18. Khojastehpour L, Afsa M, Dabbaghmanesh MH. Evaluation of Correlation between Width and Morphology of Mandibular Inferior Cortex in Digital Panoramic Radiography and Postmenopausal Osteoporosis. Iran Red Crescent Med J. 2011;13(3):1816. 